\title{
Simulating the effect of weak measurements by a phase damping channel and determining different measures of bipartite correlations in nuclear magnetic resonance
}

\author{
Akanksha Gautam $^{\mathrm{a}}$, Varad R. Pande ${ }^{\mathrm{b}}$, Amandeep Singh ${ }^{\mathrm{a}, \mathrm{c}}$, Kavita Dorai ${ }^{\mathrm{a}}$, Arvind $^{\mathrm{a}, 1, *}$ \\ ${ }^{a}$ Department of Physical Sciences, Indian Institute of Science Education 8 Research Mohali, Sector 81 SAS Nagar, Manauli PO 140306 \\ Punjab India. \\ ${ }^{b}$ Department of Physics, University of Maryland, Baltimore County (UMBC), Baltimore, Maryland 21250, USA. \\ ${ }^{c}$ Shenzhen Institute for Quantum Science and Engineering, and Department of Physics, Southern University of Science and Technology, \\ Shenzhen 518055, China
}

\section{Abstract}

Quantum discord is a measure based on local projective measurements which captures quantum correlations that may not be fully captured by entanglement. A change in the measurement process, achieved by replacing rank-one projectors with a weak positive operator-valued measure (POVM), allows one to define weak variants of quantum discord. In this work, we experimentally simulate the effect of a weak POVM on a nuclear magnetic resonance quantum information † processor. The two-qubit system under investigation is part of a three-qubit system, where one of the qubits is used as an ancillary to implement the phase damping channel. The strength of the weak POVM is controlled by varying the strength of the phase damping channel. We experimentally observed two weak variants of quantum discord namely, super quantum discord and weak quantum discord, in two-qubit Werner and Bell-diagonal states. The resultant dynamics of the states is investigated as a function of the measurement strength.

Keywords: NMR quantum information processing, Quantum discord, Weak measurement, Weak positive operator-valued measure (POVM), Phase damping channel, Super quantum discord, Weak quantum discord PACS: 03.65.Ud, 03.67.Mn

\section{Introduction}

Quantum correlations play an important role in quantum communication and quantum information processing [1]. While quantum entanglement was discovered early on by Schrödinger [2], Ollivier and Zurek [3] and Henderson and Vedral [4], independently pointed out that a different type of quantum correlations can exist in bipartite systems. The measure to quantify such correlations is termed as quantum discord (QD) $[3,4]$. The evaluation of $\mathrm{QD}$ is computationally a hard task as it involves numerical optimization and hence, alternative measures have been proposed such as geometric quantum discord [5], Gaussian geometric discord in continuous variable systems $[6]$ and relative entropy of discord [7]. The presence of nonclassical correlations in bipartite states was measured experimentally on a nuclear magnetic resonance (NMR) setup [8], witnessed via a single-shot experiment [9] and its preservation has also been explored using NMR [10]. Several

\footnotetext{
* Corresponding Author

Email addresses: akankshagautam@iisermohali.ac.in (Akanksha Gautam), varadrpande@gmail.com (Varad R. Pande), singh@sustech.edu.cn (Amandeep Singh),

${ }^{1}$ Tel \& Fax:+91-172-2240266

recent studies have explored the advantage of nonclassical correlations in quantum information processing even if the state has almost null entanglement [11, 12, 13].

In classical information theory, the mutual information between two random variables has two equivalent expressions which can be computed from the respective Shannon entropies. In contrast, for quantum mutual information, these two definitions give rise to different values as one of the expressions requires the von Neumann entropy conditioned by projective measurements. QD is defined as the difference between total mutual information and classical mutual information, wherein classical mutual information is found by determining the possible information gain about one subsystem while measuring the other subsystem via projective measurements [4]. What if one replaces these projective measurements by weak measurements? The crux of a weak measurement lies in the weak interaction between the system to be measured and the measuring apparatus. While originally the weak measurements were used in the context of weak values [14], it was later realized that any projective measurement can be obtained by a sequence of weak measurements [15]. These ideas have been used in several applications including the observation of spin Hall effect of light [16], direct measurement of single photon wavefunction [17], protection 
of quantum entanglement from decoherence [18], feedback control of a quantum system [19], to amplify small transverse deflections of an optical beam in a Sagnac interferometer [20] and for quantum state tomography [21, 22, 23].

Weak measurements allow us to define two different variants of quantum discord, which stem from the fact that QD can be defined in two mathematically equivalent ways [24]. Weak measurements have been utilized in a new definition of bipartite correlations through a weak variant of QD termed super quantum discord (SQD) [25, 26], which is numerically greater than QD. Since then SQD has attracted interest in various contexts in quantum information processing [27, 28, 29, 30]. Recently, the question of whether weak measurements can be used to gain more information about correlations present in a quantum system, was explored by formulating another weak variant of QD termed weak quantum discord (WQD), which never exceeds QD [31, 32]. While projective measurements result in a loss of coherence, the precise relationship between decoherence and measurement needs to be clarified. Simulations of the phase damping (PD) channel have been realized as projective measurements in NMR [33] and using this method quantum teleportation and the quantum Zeno effect have been implemented in an NMR setup [34].

In this work we experimentally implement the effect of a weak POVM via a phase damping channel, in two-qubit states. The PD channel is a non-unitary operation used to model the decoherence process [1]. We have also investigated the experimental behavior of two weak variants of QD namely, SQD and WQD. The determination of SQD and WQD from a bipartite state requires a weak measurement on one subsystem instead of a projective measurement. The result of a weak measurement on a Belldiagonal state and on a Werner state while finding SQD and WQD, affects the state in the same manner as if a PD channel was acting on it. We mapped the weak measurement to a PD channel and the weak measurement strength is controlled by tuning the strength of the PD channel. We use three nuclear spins to encode three qubits, where the two qubits are used to prepare two-qubit states and the third qubit acts as an ancillary qubit used to simulate a PD channel acting on one of the qubit of two-qubit states [35]. This study reports the first experimental implementation of controlled weak measurements on an NMR hardware. We successfully demonstrated that both SQD and WQD approach QD as the measurement strength is increased.

This article is organized as follows: Sec. 2.1 describes nonclassical correlations as quantified by QD. Sec. 2.2 and Sec. 2.2.2 describes weak measurement and its application in determining the SQD and WQD respectively, in twoqubit states. Sec. 3 details the mapping of weak measurement to the PD channel and Sec. 4 contains experimental results of implementing a PD channel to observe SQD and WQD. Sec. 5 contains some concluding remarks.

\section{Quantum Discord and Weak Measurements}

\subsection{Quantum Discord}

It was independently noted by Ollivier and Zurek [3] and by Henderson and Vedral [4] that certain types of mixed separable states have zero entanglement, yet contain nonclassical correlations. Mathematically, QD captures these correlations and is the difference between the total correlation $I\left(\rho_{A B}\right)$ and the classical correlation $J\left(\rho_{A \mid B}\right)$ :

$$
\begin{aligned}
I\left(\rho_{A B}\right) & =S\left(\rho_{A}\right)+S\left(\rho_{B}\right)-S\left(\rho_{A B}\right) \\
J\left(\rho_{A \mid B}\right) & =S\left(\rho_{A}\right)-S\left(\rho_{A \mid B}\right)
\end{aligned}
$$

where $S\left(\rho_{A B}\right)=-\operatorname{Tr}\left(\rho_{A B} \log _{2} \rho_{A B}\right)$ is the von Neumann entropy of the quantum state, $\rho_{A B}$ is shared between two parties $A$ and $B, \rho_{A, B}=\operatorname{Tr}_{B, A}\left(\rho_{A B}\right)$ is the reduced density operator of a subsystem, and $S\left(\rho_{A \mid B}\right)$ is the conditional von Neumann entropy of subsystem $A$ when subsystem $B$ has already been measured. Quantum discord is then defined as:

$$
Q D\left(\rho_{A B}\right)=I\left(\rho_{A B}\right)-\max J\left(\rho_{A \mid B}\right)
$$

where the maxima is taken over all possible projective measurements on the subsystem $B$.

It turns out that we can also write quantum discord in a mathematically equivalent form [24]:

$$
Q D\left(\rho_{A B}\right)=I\left(\rho_{A B}\right)-\max I\left(\rho_{A B}^{\prime}\right)
$$

where $\rho_{A B}^{\prime}$ is the density operator of the combined system after a projective measurement over subsystem $B$ has been carried out. As has been discussed in a lucid manner in [32] this alternative mathematical form that was originally introduced for defining QD for multipartite systems, also leads to an alternative interpretation of QD and is useful in generalizing QD to the weak measurement scenario.

For a two-qubit system, projective measurements on the single-qubit subsystem $B$ can be characterized by the Bloch sphere direction $\theta, \phi$. The corresponding projectors $\Pi_{1}^{\theta, \phi}$ and $\Pi_{2}^{\theta, \phi}$ can be constructed utilizing two orthogonal vectors as follows:

$$
\begin{gathered}
|\psi\rangle_{1}^{\theta, \phi}=\cos \frac{\theta}{2}|0\rangle+e^{i \phi} \sin \frac{\theta}{2}|1\rangle \\
|\psi\rangle_{2}^{\theta, \phi}=-\sin \frac{\theta}{2}|0\rangle+e^{i \phi} \cos \frac{\theta}{2}|1\rangle \\
\Pi_{1}^{\theta, \phi}=|\psi\rangle_{1}^{\theta, \phi}\left\langle\left.\psi\right|_{1} ^{\theta, \phi} \text { and } \Pi_{2}^{\theta, \phi}=\mid \psi\right\rangle_{2}^{\theta, \phi}\left\langle\left.\psi\right|_{2} ^{\theta, \phi} .\right.
\end{gathered}
$$

The state of the subsystem $A$ after a projective measurement on subsystem $B$ gives a positive result for the projector $\Pi_{j}^{\theta, \phi}$ and can be written as:

$$
\rho_{A \mid \Pi_{j}^{\theta, \phi}}=\frac{1}{p_{j}} \operatorname{Tr}_{B}\left[\left(I \otimes \Pi_{j}^{\theta, \phi}\right) \rho_{A B}\left(I \otimes \Pi_{j}^{\theta, \phi}\right)\right]
$$

where $p_{j}$ is the probability of the measurement outcome corresponding to the projectors $\Pi_{j}^{\theta, \phi}$. The state of the 
combined system after a measurement on the system $B$ can be written as:

$$
\rho_{A B}^{\prime}=\sum_{j=1}^{2}\left[\left(I \otimes \Pi_{j}^{\theta, \phi}\right) \rho_{A B}\left(I \otimes \Pi_{j}^{\theta, \phi}\right)\right] .
$$

The conditional von Neumann entropy required in Eq. (1) is given by:

$$
S\left(\rho_{A \mid B}\right)=\sum_{j=1}^{2} p_{j} S\left(\rho_{A \mid \Pi_{j}^{\theta, \phi}}\right) .
$$

QD can be computed by utilizing the above defined projectors in Eq.(7), followed by substituting the parameterized conditional entropy into Eq.(1):

$$
\begin{aligned}
Q D\left(\rho_{A B}\right) & =I\left(\rho_{A B}\right)-\max _{\{\theta, \phi\}} J\left(\rho_{A \mid B}\right) \\
& =I\left(\rho_{A B}\right)-\max _{\{\theta, \phi\}} I\left(\rho_{A B}^{\prime}\right) .
\end{aligned}
$$

Thus one can compute QD in two different ways: by maximizing the $J\left(\rho_{A \mid B}\right)$ or by maximizing $I\left(\rho_{A B}^{\prime}\right)$ (which for projective measurements turn out to be the same) over $\theta \in[0, \pi]$ and $\phi \in[0,2 \pi]$, and substituting $\theta$ and $\phi$ back into Eqs. (8a) \& (8b).

\subsection{Weak Variants of Quantum Discord}

What happens if we replace the projective measurement used to compute QD by a weak measurement? Weak measurements, where the system-apparatus interaction is weak, extract limited information from the system and correspondingly disturb the system in a limited way. Repeated weak measurements lead to a strong or a projective measurement. The positive operator valued measure (POVM) corresponding to the weak measurement is defined through the operators:

$$
\begin{gathered}
P(x)=\sqrt{\frac{1-\tanh x}{2}} \Pi_{\psi_{1}}^{\theta, \phi}+\sqrt{\frac{1+\tanh x}{2}} \Pi_{\psi_{2}}^{\theta, \phi} \\
P(-x)=\sqrt{\frac{1+\tanh x}{2}} \Pi_{\psi_{1}}^{\theta, \phi}+\sqrt{\frac{1-\tanh x}{2}} \Pi_{\psi_{2}}^{\theta, \phi}
\end{gathered}
$$

where the strength of the weak measurement is parameterized by the real parameter $x \geq 0$. The POVM operators satisfy $P(x)^{\dagger} P(x)+P(-x)^{\dagger} P(-x)=I$. For $x=0, P(0)$ reduces to $\frac{1}{\sqrt{2}} I i$. e. no measurement at all and in the case of $x \rightarrow \infty, P(x)$ and $P(-x)$ reduce to the projectors $\Pi_{\psi_{2}}^{\theta, \phi}$ and $\Pi_{\psi_{1}}^{\theta, \phi}$, respectively (as defined in Eq. (4)).

After the weak measurement, the state of the combined system becomes:

$$
\begin{aligned}
\rho_{A B}^{x}=(I \otimes P(x)) \rho_{A B}(I \otimes P(x))+ \\
(I \otimes P(-x)) \rho_{A B}(I \otimes P(-x))
\end{aligned}
$$

The post-measurement state of subsystem $A$ for each outcome can be written as

$$
\rho_{A \mid P( \pm x)}=\frac{1}{p( \pm x)} \operatorname{Tr}_{B}\left[(I \otimes P( \pm x)) \rho_{A B}(I \otimes P( \pm x))\right]
$$

Where $p( \pm x)$ are the probabilities for $P( \pm x)$. The question now is: how can we define weak variants of quantum discord?

\subsubsection{Super quantum discord}

If we take Eq. (8a) as the basic definition of QD and replace the projective measurement with a weak POVM (as defined above), we obtain a straightforward generalization of QD. This can be written down by first computing the conditional entropy and classical information as:

$$
\begin{aligned}
& S_{x}\left(\rho_{A \mid B}\right)=p(x) S_{x}\left(\rho_{A} \mid P(x)\right)+p(-x) S_{x}\left(\rho_{A} \mid P(-x)\right) \\
& J_{x}\left(\rho_{A \mid B}\right)=S\left(\rho_{A}\right)-S_{x}\left(\rho_{A \mid B}\right) .
\end{aligned}
$$

In terms of the above, a weak variant of quantum discord can be defined which is called super quantum discord (SQD):

$$
S Q D\left(\rho_{A B}\right)=I\left(\rho_{A B}\right)-\max _{\{\theta, \phi\}} J_{x}\left(\rho_{A \mid B}\right)
$$

which depends on the measurement strength $x$. The value of SQD is always greater than QD because $S_{x}\left(\rho_{A \mid B}\right)$ is always larger than $S\left(\rho_{A \mid B}\right)$, as the weak measurement is performed on subsystem $B$ while disturbing the state weakly, and reveals less information about the subsystem $A$. In the limiting case of no measurement performed on system $B$ at all, $S_{x}\left(\rho_{A \mid B}\right) \rightarrow S\left(\rho_{A}\right)$ and SQD discord will be equal to the total correlations.

\subsubsection{Weak Quantum Discord}

SQD has the feature that it is always larger than QD and has hence been found somewhat dissatisfying. A weak generalization of QD is possible if we take Eq. (8b) to be a fundamental definition of $\mathrm{QD}$ and replace the second term with its weak equivalent [24]. This process allows us to define another weak variant of quantum discord called weak quantum discord (WQD), which for a two-qubit system can be written as:

$$
W Q D\left(\rho_{A B}\right)=I\left(\rho_{A B}\right)-\max _{\{\theta, \phi\}} I\left(\rho_{A B}^{x}\right)
$$

where $\rho_{A B}^{x}$ is the density operator of the composite system after a measurement of strength $x$ has been performed on the subsystem $B$ as given in Eq. (10). WQD as a quantifier has the nice property that it is always less than QD and in the limit $x \rightarrow 0$ it approaches 0 , while for $x \rightarrow \infty$ it approaches QD. In the strong measurement limit, both SQD and WQD become the same and are equal to QD. The key observation which plays a role in these two different generalizations of $\mathrm{QD}$ in the weak regime, is that the two different equivalent expressions for $\mathrm{QD}$ do not remain the same in the weak measurement regime. For interesting and more detailed interpretations of SQD and WQD, the reader is referred to Ref. [32]. 


\section{Simulating Weak POVM Via a Phase Damping Channel}

A projective measurement collapses the state, thereby killing all the off-diagonal terms (coherences) in the density matrix, in the measurement basis [34]. The weak measurement formalism, as described by Aharonov, Albert and Vaidmann (AAV) [14] utilizes the weak interaction [36]. The weak interaction couples the system weakly with the measuring device, and therefore the state of the system retains its coherence partially, even after the measurement. Later, Oreshkov and Brun [15] showed that any generalized measurement can be modeled by a sequence of weak POVMs and for the two-qubit case are given in Eq.(9).

We consider two types of states to investigate the behavior of the quantities SQD and WQD (defined in the previous section) with respect to measurement strength and to compare it with QD, namely, the Werner states and Bell-diagonal states. The two-qubit Werner states are defined as:

$$
\rho_{A B}^{w s}=z\left|\psi^{-}\right\rangle\left\langle\psi^{-}\right|+\frac{1}{4}(1-z) I
$$

where $1-z$ quantifies the amount of mixedness, $0 \leq z \leq 1$ and $\left|\psi^{-}\right\rangle=\frac{1}{\sqrt{2}}(|01\rangle-|10\rangle)$. The two-qubit Bell-diagonal states [37] are defined as:

$$
\rho_{A B}^{b s}=\frac{1}{4}\left[I \otimes I+\sum_{i=1}^{3} c_{i}\left(\sigma_{i} \otimes \sigma_{i}\right)\right]
$$

where $\left(\sigma_{1}, \sigma_{2}, \sigma_{3}\right)$ are the Pauli matrices and $-1 \leq c_{1}, c_{2}, c_{3} \leq$ 1.

The evaluation of SQD and WQD in both the states involves an optimization over all possible projectors by varying $\theta \in[0, \pi]$ and $\phi \in[0,2 \pi]$ as given in Eq.(13). The optimization gives the highest possible classical correlations at $\theta=\pi$ and $\phi=\pi$ for the Werner states as well for the Bell-diagonal states. On substituting the optimal values of $\theta$ and $\phi$ into Eq.(9), the weak POVMs get simplified to:

$$
\begin{array}{r}
P(x)=\sqrt{\frac{1-\tanh x}{2}}|0\rangle\left\langle 0\left|+\sqrt{\frac{1+\tanh x}{2}}\right| 1\right\rangle\langle 1| \\
P(-x)=\sqrt{\frac{1+\tanh x}{2}}|0\rangle\left\langle 0\left|+\sqrt{\frac{1-\tanh x}{2}}\right| 1\right\rangle\langle 1| .
\end{array}
$$

A single-qubit mixed state $\rho$ on the Bloch sphere can be expressed as:

$$
\rho=\frac{1}{2}\left(I+r_{x} \sigma_{1}+r_{y} \sigma_{2}+r_{z} \sigma_{3}\right)
$$

where $r_{x}, r_{y}$ and $r_{z}$ are the coordinates of the Bloch vector and $I$ is the $2 \times 2$ identity matrix. The effect of the simplified weak POVM given in Eq.(17) on the single-qubit state $\rho$ can be readily computed and in the matrix form is written as:

$$
\begin{aligned}
\rho & =\frac{1}{2}\left[\begin{array}{cc}
1+r_{z} & r_{x}-i r_{y} \\
r_{x}+i r_{y} & 1-r_{z}
\end{array}\right] \\
\Downarrow & \\
\rho_{w m}^{\prime} & =\frac{1}{2}\left[\begin{array}{cc}
1+r_{z} & \left(r_{x}-i r_{y}\right) \operatorname{sech} x \\
\left(r_{x}+i r_{y}\right) \operatorname{sech} x & 1-r_{z} .
\end{array}\right]
\end{aligned}
$$

It is clear from the post weak-measurement state $\rho_{w m}^{\prime}$ that the off-diagonal terms are a monotonically decreasing function of the measurement strength $x$, leading to decoherence. The extent to which the weak measurement decoheres the state $\rho$ depends on the measurement strength $x$.

We now turn to the PD channel, which causes loss of coherence and leads to the decay of the off-diagonal terms of the density matrix and can be described by a completely positive trace preserving map described through the Kraus operators [38]:

$$
\begin{gathered}
\rho_{P D}^{\prime}=E_{0} \rho E_{0}^{\dagger}+E_{1} \rho E_{1}^{\dagger} \\
E_{0}=\frac{1+\sqrt{1-\lambda}}{2} I+\frac{1-\sqrt{1-\lambda}}{2} \sigma_{3} \\
E_{1}=\frac{\sqrt{\lambda}}{2} I-\frac{\sqrt{\lambda}}{2} \sigma_{3}
\end{gathered}
$$

where the parameter $\lambda \in[0,1]$ represents the strength of the PD channel. The action of the PD channel on a general one-qubit state in the matrix form is given by:

$$
\begin{aligned}
\rho & =\frac{1}{2}\left[\begin{array}{cc}
1+r_{z} & r_{x}-i r_{y} \\
r_{x}+i r_{y} & 1-r_{z}
\end{array}\right] \\
\Downarrow & \\
\rho_{P D}^{\prime} & =\frac{1}{2}\left[\begin{array}{cc}
1+r_{z} & \left(r_{x}-i r_{y}\right) \sqrt{1-\lambda} \\
\left(r_{x}+i r_{y}\right) \sqrt{1-\lambda} & 1-r_{z} .
\end{array}\right]
\end{aligned}
$$

The effect of the PD channel is similar to the weak POVM on a single-qubit state, wherein the off-diagonal terms are diminished. Since both 'sech $x$ ' and ' $\sqrt{1-\lambda}$ ' are monotonically decreasing functions, they can be mapped onto each other with an appropriate scaling factor. Therefore, the action of the PD channel is in one-to-one correspondence with weak POVM described in Eq. (9).

In order to implement the PD channel we follow an indirect approach [35], wherein non-unitary operators can be thought of as unitary operations on an extended quantum system built upon the Duality Quantum Computing (DQC) framework [39]. This framework requires an ancillary qubit. It has been demonstrated [35] that the Kraus operator $E_{k}$ describing the non-unitary transformation corresponding to the PD channel can be efficiently implemented on a qubit if the unitary operators $V, W$, $U_{0}$ and $U_{1}$ can be found in the two-qubit space, where $V$ and $W$ act on the ancilla qubit, and $U_{0}$ and $U_{1}$ act on the target qubit controlled by the ancilla qubit. The operators need to satisfy:

$$
E_{k}(k=0,1)=\sum_{i=0}^{1} W_{k i} V_{i 0} U_{i}
$$




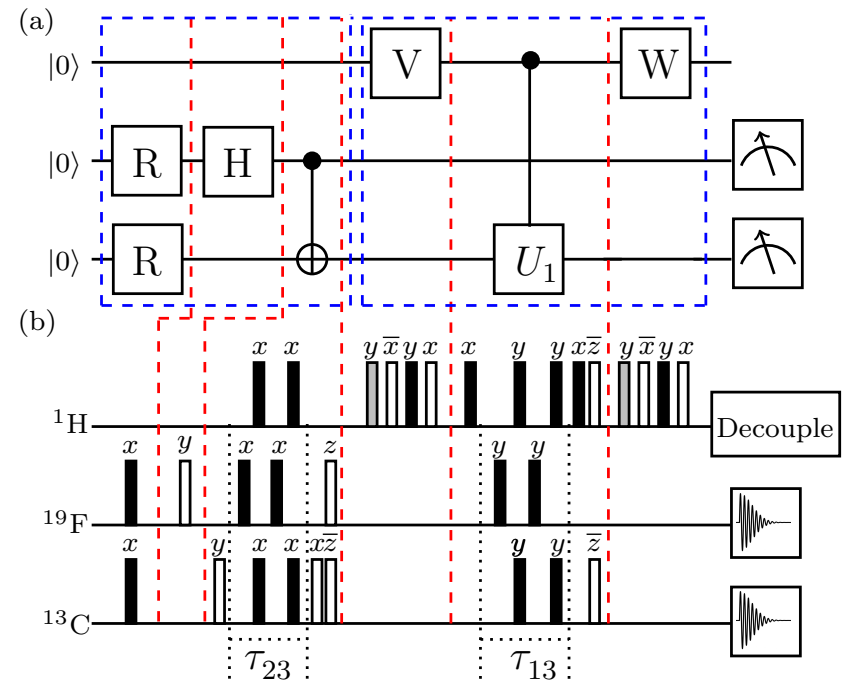

Figure 1: (a) The quantum circuit in the left block creates a Belldiagonal state and a Werner state $\left(\rho_{0} \otimes \rho_{\psi}\right)$ on two qubits $\left(\rho_{\psi}\right)$, with the third qubit $\left(\rho_{0}\right)$ acting as ancillary; $\mathrm{H}$ denotes a Hadamard gate, $\mathrm{R}$ is a NOT gate in the case of the Werner state and $I_{2}$ identity operation (no operation) in the case of the Bell-diagonal state. The quantum circuit in the right block implements a phase damping channel on one of the qubits of hence prepared two-qubit state using an ancillary qubit. (b) NMR pulse sequences corresponding to the quantum circuits where the unfilled rectangles denote $\frac{\pi}{2}$ radiofrequency (rf) pulses, the filled rectangles denote $\pi$ rf pulses and the shaded rectangles denote $\theta$ rf pulses where $\theta=-2 \sin ^{-1} \sqrt{\frac{1-\sqrt{1-\lambda}}{2}}$, and $\lambda$ is the strength of the PD channel, lying between 0 and 1 . The phase of the rf pulse is given above each pulse and a bar over a phase represents negative phase. The free evolution time intervals $\tau_{12}$ and $\tau_{23}$ are given by $1 /\left(2 \mathrm{~J}_{12}\right)$ and $1 /\left(2 \mathrm{~J}_{23}\right)$ respectively, where $\mathrm{J}_{\mathrm{ij}}$ represents the scalar coupling strength between qubits $i$ and $j$.

where $W_{k i}$ is $k i$ th element of $W$ operator, $V_{i 0}$ is an element of the first column of $V$ operator and $U_{i}$ is the controlled operator. A comparison with the decomposition of Kraus operators of the PD channel is given in Eq.(20). The unitary operators $V, W, U_{0}$ and $U_{1}$ can be evaluated as:

$$
\begin{aligned}
& U_{0}=I, \quad U_{1}=\sigma_{3}, \\
& V=W=\frac{1}{2}\left[\begin{array}{cc}
\sqrt{\frac{1+\sqrt{1-\lambda}}{2}} & \sqrt{\frac{1-\sqrt{1-\lambda}}{2}} \\
\sqrt{\frac{1-\sqrt{1-\lambda}}{2}} & -\sqrt{\frac{1+\sqrt{1-\lambda}}{2}}
\end{array}\right] .
\end{aligned}
$$

The quantum circuit to implement the PD channel is shown in Fig. 1(a), where Werner states and Bell-diagonal states (Eq.(15-16)) states are created on qubits 2 and 3, qubit 1 acts as an ancilla, and the the PD channel acts on qubit 3. The strength of the PD channel is controlled by the $V$ gate. The effect of the PD channel can be evaluated by tracing out the ancillary qubit. Physically this was achieved by performing the measurements on the state of the two-qubit subsystem consisting of qubits 2 and 3, while ignoring the qubit 1 , as is shown in Fig. 1.

We are now ready to experimentally investigate the behavior of SQD and WQD by varying the measurement strength. It is important to mention here that in an NMR set up the measurement is already weak (termed as an (a)

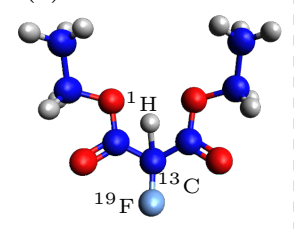

$\nu_{\mathrm{H}}=3331.50 \mathrm{~Hz}$

$\nu_{\mathrm{F}}=-1110992.88 \mathrm{~Hz}$

$\nu_{\mathrm{C}}=12891.11 \mathrm{~Hz}$

$\mathrm{J}_{\mathrm{HF}}=47.5 \mathrm{~Hz}$

$\mathrm{J}_{\mathrm{HC}}=161.6 \mathrm{~Hz}$

$\mathrm{J}_{\mathrm{FC}}=-191.8 \mathrm{~Hz}$

$\mathrm{T}_{1}^{\mathrm{H}}=4.10 \pm 0.06 \mathrm{~s}$

$\mathrm{T}_{1}^{\mathrm{F}}=6.30 \pm 0.06 \mathrm{~s}$

$\mathrm{T}_{1}^{\mathrm{C}}=6.74 \pm 0.17 \mathrm{~s}$

$\mathrm{T}_{2}^{\mathrm{H}}=1.18 \pm 0.12 \mathrm{~s}$

$\mathrm{T}_{2}^{\mathrm{F}}=2.99 \pm 0.10 \mathrm{~s}$

$\mathrm{T}_{2}^{\mathrm{C}}=3.37 \pm 0.19 \mathrm{~s}$

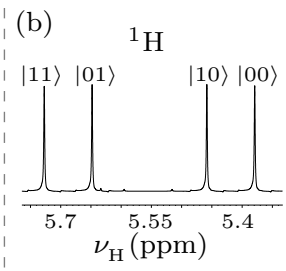

(c)

${ }^{1} \mathrm{H}$
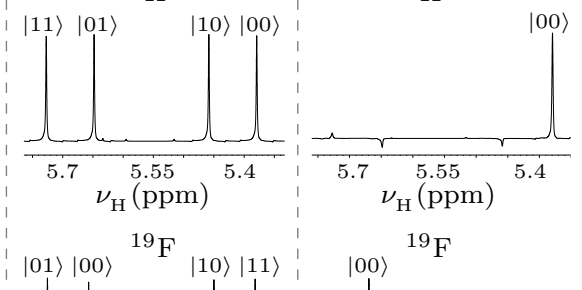

$|00\rangle$

${ }^{19} \mathrm{~F}$
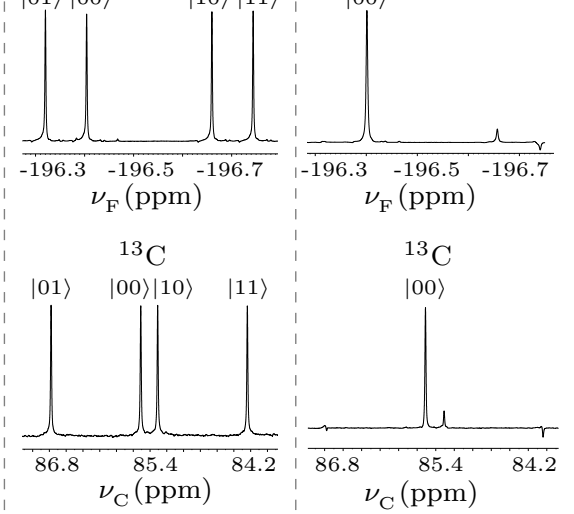

Figure 2: (a) Molecular structure of ${ }^{13} \mathrm{C}$-labeled diethylfluoromalonate with the three qubits labeled as ${ }^{1} \mathrm{H},{ }^{19} \mathrm{~F}$ and ${ }^{13} \mathrm{C}$. NMR parameters i.e. the chemical shift $\nu_{i}$ (in $\mathrm{Hz}$ ) of each nuclear spin, spinspin coupling between them $\mathrm{J}_{i j}$ (in $\mathrm{Hz}$ ), spin-lattice relaxation times $\mathrm{T}_{1}^{i}$ and spin-spin relaxation times $\mathrm{T}_{2}^{i}$ (in seconds). NMR spectrum of (b) thermal equilibrium state obtained after a $\frac{\pi}{2}$ readout pulse and (c) pseudopure state. The resonance lines of each qubit in the spectra are labeled by the corresponding logical states of the other qubits.

ensemble weak measurement) since the interaction of the measuring rf coil with the nuclear spins is weak [33]. However, we are not using that weak measurement here. Our weak measurement is simulated in a controlled way by the PD channel, which is implemented with the help of the ancilla qubit.

\section{Experimental Implementation of a Weak POVM}

As discussed earlier in Sec. 3, weak measurements can be mapped onto a PD channel and the strength of the weak measurement can be varied by tuning the strength of the PD channel. For the experimental realization on an NMR quantum processor, we realize the three qubits as the three spin-1/2 nuclei of ${ }^{13} \mathrm{C}$-labeled diethyl fluoromalonate dissolved in acetone-D6. The ${ }^{1} \mathrm{H},{ }^{19} \mathrm{~F}$ and ${ }^{13} \mathrm{C}$ nuclear spins are labeled as the first, second and third qubit, respectively. It should be noted here that two-qubit system was simulated by ${ }^{19} \mathrm{~F}$ and ${ }^{13} \mathrm{C}$ nuclear spins while ${ }^{1} \mathrm{H}$ spin was utilized as the ancillary qubit. The molecular structure along with relevant experimental parameters and corresponding NMR spectrum of the thermal equilibrium state are shown in Figs. 2(a) and (b) respectively. The Hamiltonian for a three-qubit system in a rotating frame under 

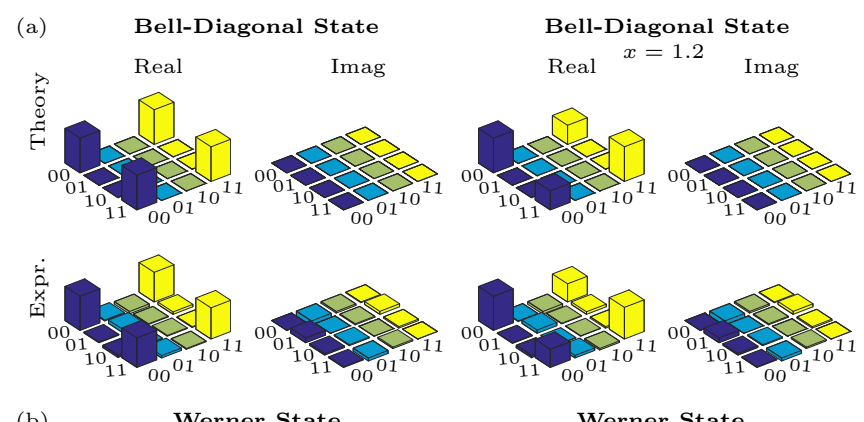

(b)
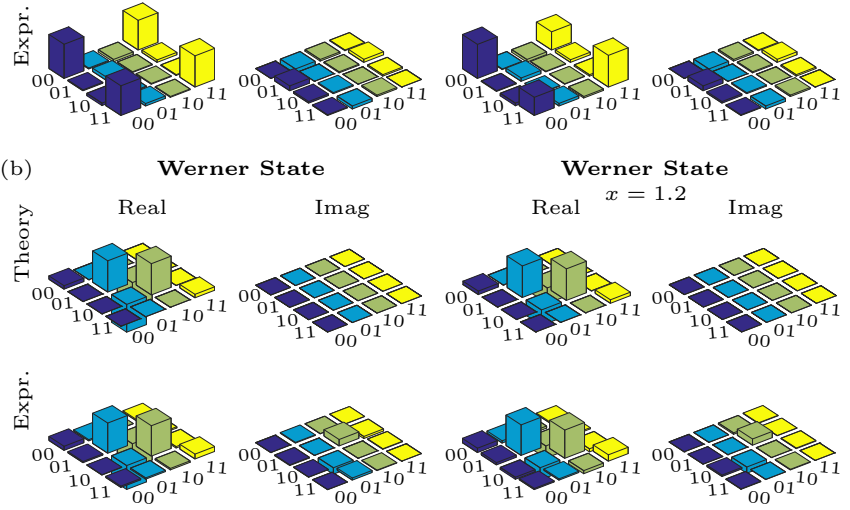

Figure 3: Real and imaginary parts of theoretically expected and the experimentally reconstructed tomographs of (a) Bell-diagonal state and (b) Werner state before (left) and after (right) PD channel implementation at a measurement strength $x=1.2$.

the weak approximation [40] is given by:

$$
H=-\sum_{i=1}^{3} \nu_{i} I_{z}^{i}+\sum_{i>j, i=1}^{3} \mathrm{~J}_{i j} I_{z}^{i} I_{z}^{j}
$$

where $i, j=1,2$ and 3 labels the qubit, $v_{i}$ represents the chemical shift of the respective nuclei, $\mathrm{J}_{i j}$ is the scalar coupling constant between the $i^{t h}$ and $j^{\text {th }}$ nuclear spins and $I_{z}^{i}$ denotes the $z$ component of the spin angular momentum operators for the $i^{\text {th }}$ nucleus. We used the spatial averaging technique $[41,42]$ to achieve the initial three-qubit pseudopure state (PPS) $|000\rangle$ from the thermal equilibrium state, with the density operator $\rho_{000}$ given by:

$$
\rho_{000}=\frac{(1-\epsilon)}{8} I+\epsilon|000\rangle\langle 000|
$$

where $\epsilon \sim 10^{-5}$ represents the spin polarization at room temperature and $I$ is the $8 \times 8$ identity operator. The identity operator does not evolve and the measurable NMR signal can be attributed to the deviation density matrix. The NMR spectrum of the three-qubit PPS is shown in Fig. 2(c). The experimentally prepared PPS was tomographed using full quantum state tomography [43]. The state fidelity was found to be $0.981 \pm 0.006$ and was computed using the Uhlmann-Jozsa measure [44, 45]:

$$
\mathrm{F}=\left[\operatorname{Tr}\left(\sqrt{\sqrt{\rho_{\mathrm{th}}} \rho_{\mathrm{ex}} \sqrt{\rho_{\mathrm{th}}}}\right)\right]^{2}
$$

where $\rho_{\text {th }}$ and $\rho_{\text {ex }}$ denote the theoretical and experimental density operators, respectively and $\mathrm{F}$ is normalized using $\mathrm{F} \rightarrow 1$ as $\rho_{\mathrm{ex}} \rightarrow \rho_{\text {th }}$. All the experimental density matrices were reconstructed by performing full quantum state tomography [43] using a set of seven preparatory pulses $\{I I I, X X X, I I Y, X Y X, Y I I, X X Y, I Y Y\}$, where $I$ represents 'no-operation' and $X(Y)$ denotes local $\frac{\pi}{2}$ unitary rotation with phase $x(y)$ which is implemented by applying a spin-selective $\frac{\pi}{2}$ pulse. We performed all the experiments at room temperature $293 \mathrm{~K}$ on a Bruker Avance-III 600 $\mathrm{MHz}$ FT-NMR spectrometer equipped with a QXI probe.

The quantum circuit to implement a weak measurement, simulated by a PD channel, is shown in Fig. 1(a). The left block in the circuit creates a Werner or a Belldiagonal state $\left(\rho_{0} \otimes \rho_{\psi}\right)$ on two qubits $\left(\rho_{\psi}\right)$ with the third qubit $\left(\rho_{0}\right)$ acting as ancillary; $\mathrm{R}$ is a NOT gate in the case of the Werner state and a 'no-operation' for a Bell-diagonal state. The right block in the circuit depicts a PD channel acting on the one of the qubits of two-qubit state using an ancillary qubit, and the strength of the PD channel being controlled by a local rotation achieved by the $V$ gate acting on the ancillary qubit.

Table 1: Experimental (Exp) results of weak measurement strength $x$ varied in a Werner (W) and a Bell-diagonal (BD) state while implementing the PD channel.

\begin{tabular}{ccc}
\hline \hline Theory $x$ & $\operatorname{Exp} x(\mathrm{~W})$ & $\operatorname{Exp} x(\mathrm{BD})$ \\
\hline \hline 0.00 & $0.091 \pm 0.047$ & $0.069 \pm 0.036$ \\
0.34 & $0.503 \pm 0.018$ & $0.373 \pm 0.002$ \\
0.55 & $0.666 \pm 0.015$ & $0.548 \pm 0.005$ \\
0.75 & $0.831 \pm 0.016$ & $0.721 \pm 0.005$ \\
0.95 & $1.016 \pm 0.016$ & $0.907 \pm 0.004$ \\
1.20 & $1.215 \pm 0.020$ & $1.103 \pm 0.007$ \\
1.50 & $1.479 \pm 0.024$ & $1.350 \pm 0.007$ \\
1.75 & $1.819 \pm 0.045$ & $1.667 \pm 0.018$ \\
2.00 & $2.122 \pm 0.046$ & $1.937 \pm 0.018$ \\
2.50 & $2.454 \pm 0.062$ & $2.213 \pm 0.026$ \\
3.00 & $3.242 \pm 0.151$ & $2.795 \pm 0.042$ \\
3.50 & $3.758 \pm 0.095$ & $3.448 \pm 0.069$ \\
4.00 & $4.107 \pm 0.167$ & $4.259 \pm 0.123$ \\
4.50 & $4.402 \pm 0.062$ & $4.575 \pm 0.069$ \\
5.00 & $4.839 \pm 0.137$ & $5.030 \pm 0.180$ \\
\hline \hline
\end{tabular}

Experimentally, we prepared a Werner state with mixedness strength $z=0.8$. The second term on the RHS of Eq.(15) is a singlet state and was created experimentally, followed by quantum state tomography (QST). To obtain the Werner state of a desired $z$ value the QST generated singlet state was numerically added to the identity and thus, a Werner state with fidelity $0.990 \pm 0.001$ was created. Next the Bell-diagonal state with $c_{1}=1$, $c_{2}=-1$ and $c_{3}=1$ was prepared experimentally with fidelity $0.980 \pm 0.002$. The next step is to perform a weak measurement on the second qubit (treated as a subsystem of the two-qubit system). As described in Sec. 3, the weak measurement is simulated by the PD channel and the strength of PD channel is controlled by the real parameter $\lambda$ which is related to weak measurement strength $x$ as 
(a)

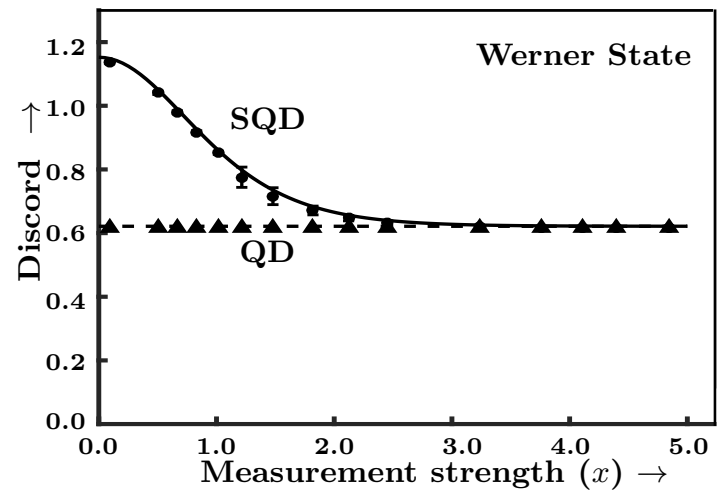

(b)

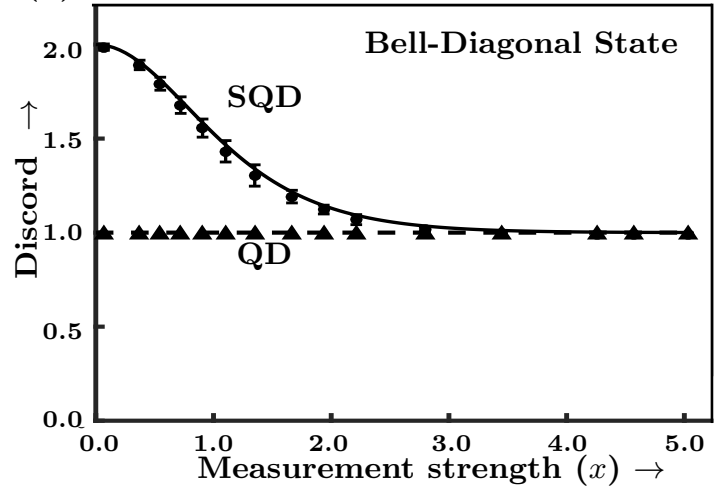

Figure 4: Plot of SQD and QD with measurement strength $(x)$ for the two-qubit experimentally prepared (a) Werner state and (b) Belldiagonal state. The solid and dashed lines represent the theoretically computed values while the filled circles and triangles represent the experimental values of SQD and QD, respectively.

$\lambda=1-\operatorname{sech}^{2} x$. We implemented the PD channel on the one of the qubits of the prepared Werner state using the ancillary qubit and the strength of the PD channel was increased corresponding to weak measurement strength $x$ as shown in Table-1. We performed a similar experiment for the Bell-diagonal states, which was directly prepared from the left block in the circuit as depicted in Fig. 1(a) and the PD channel was implemented with increasing weak measurement strength $x$ as shown in Table-1. All the experimentally prepared three-qubit states were tomographed before and after implementing PD channel. Both the twoqubit Werner and Bell-diagonal states were reconstructed utilizing QST and tracing out the ancillary qubit. The tomograph of one such experimentally reconstructed density matrix of both initially prepared states is shown on the LHS of Fig. 3(a) and Fig. 3(b), respectively. The tomographs on the RHS of Fig. 3(a) and (b) depicts the states after the action of the PD channel corresponding to the weak measurement strength $x=1.2$.

We investigated theoretically and experimentally, the behavior of SQD, WQD, and QD present in both Werner and Bell-diagonal states while increasing the measurement strength $x$, and the results are plotted in Fig. 4 and Fig. 5, respectively. Our results show that in both types of states,
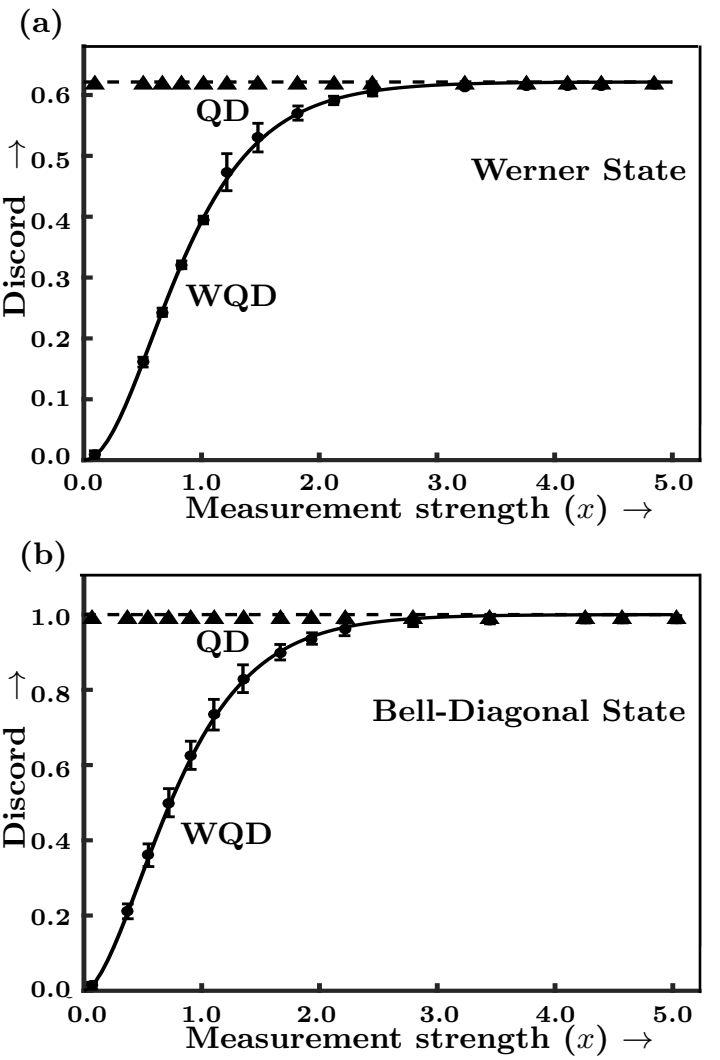

Figure 5: Plot of WQD and QD with measurement strength $(x)$ for the two-qubit experimentally prepared (a) Werner state and (b) Belldiagonal state. The solid and dashed lines represent the theoretically computed values while the filled circles and triangles represent the experimental values of WQD and QD, respectively.

the value of SQD is always greater than QD and is maximum at zero measurement strength, which implies that the measured state is undisturbed. On the other hand, as a quantifier WQD is never greater than QD and allows the researcher to navigate the region between $x \longrightarrow 0$ when no measurement is performed (and quantum correlations are intact) and $x \longrightarrow \infty$ when a projective measurement is performed (and quantum correlations are destroyed). Furthermore, as evidenced from Fig. 4 and Fig. 5, both SQD and WQD approach QD as the measurement strength increases. Our experimental results are hence in consonance with the theoretically expected behavior of the quantifiers of discord for the case of a weak measurement.

\section{Concluding Remarks}

We have implemented a weak POVM, exploiting its relationship with the PD channel, on an NMR quantum information processor. The noise induced by the PD channel has been exploited to mimic the disturbance introduced by a weak measurement process. The weak POVM was experimentally applied to find the SQD and WQD in two classes of bipartite quantum states, namely, the Belldiagonal state and the Werner state. The SQD and WQD were contrasted against QD and it was observed that both 
these quantities converge to $\mathrm{QD}$ as the strength of the measurement increases. The monotonicity of SQD and WQD was also confirmed. Our results could be useful for iterative experimental information processing protocols which seek to disturb the state only slightly. Although the interpretation of weak variants of QD remains elusive, this work opens up the possibility of further experimental investigations on such variants, which could potentially exploit correlations beyond QD [46, 47].

Declaration of Interest: The authors declare that they have no conflict of interest.

Acknowledgments All the experiments were performed on a Bruker Avance-III $600 \mathrm{MHz}$ FT-NMR spectrometer at the NMR Research Facility of IISER Mohali.

\section{References}

[1] M. A. Nielsen, I. L. Chuang, Quantum Computation and Quantum Information, Cambridge University Press, 2000.

[2] E. Schrödinger, Discussion of probability relations between separated systems, Proc. Camb. Phil. Soc. 31 (4) (1935) 555-563.

[3] H. Ollivier, W. H. Zurek, Quantum discord: A measure of the quantumness of correlations, Phys. Rev. Lett. 88 (2001) 017901.

[4] L. Henderson, V. Vedral, Classical, quantum and total correlations, Journal of Physics A: Mathematical and General 34 (35) (2001) 6899-6905.

[5] B. Dakić, V. Vedral, Č. Brukner, Necessary and sufficient condition for nonzero quantum discord, Phys. Rev. Lett. 105 (2010) 190502.

[6] G. Adesso, A. Datta, Quantum versus classical correlations in Gaussian states, Phys. Rev. Lett. 105 (2010) 030501.

[7] K. Modi, T. Paterek, W. Son, V. Vedral, M. Williamson, Unified view of quantum and classical correlations, Phys. Rev. Lett. 104 (2010) 080501.

[8] I. A. Silva, D. Girolami, R. Auccaise, R. S. Sarthour, I. S. Oliveira, T. J. Bonagamba, E. R. deAzevedo, D. O. SoaresPinto, G. Adesso, Measuring bipartite quantum correlations of an unknown state, Phys. Rev. Lett. 110 (2013) 140501.

[9] A. Singh, Arvind, K. Dorai, Witnessing nonclassical correlations via a single-shot experiment on an ensemble of spins using nuclear magnetic resonance, Phys. Rev. A 95 (2017) 062318.

[10] H. Singh, Arvind, K. Dorai, Experimentally freezing quantum discord in a dephasing environment using dynamical decoupling, EPL 118 (5) (2017) 50001.

[11] S. Pirandola, Quantum discord as a resource for quantum cryptography, Sci. Rep. 4 (5) (2014) 6956.

[12] B. Dakić, Y. O. Lipp, X. Ma, M. Ringbauer, S. Kropatschek, S. Barz, T. Paterek, V. Vedral, A. Zeilinger, Č. Brukner, P. Walther, Quantum discord as resource for remote state preparation, Nature Physics 8 (9) (2012) 666-670.

[13] K. Modi, A. Brodutch, H. Cable, T. Paterek, V. Vedral, The classical-quantum boundary for correlations: Discord and related measures, Rev. Mod. Phys. 84 (2012) 1655-1707.

[14] Y. Aharonov, D. Z. Albert, L. Vaidman, How the result of a measurement of a component of the spin of a spin- $1 / 2$ particle can turn out to be 100, Phys. Rev. Lett. 60 (1988) 1351-1354.

[15] O. Oreshkov, T. A. Brun, Weak measurements are universal, Phys. Rev. Lett. 95 (2005) 110409.

[16] O. Hosten, P. Kwiat, Observation of the spin Hall effect of light via weak measurements, Science 319 (5864) (2008) 787-790.
[17] J. S. Lundeen, B. Sutherland, A. Patel, C. Stewart, C. Bamber, Direct measurement of the quantum wavefunction, Nature 474 (7350) (2011) 188-191.

[18] Y. S. Kim, J. C. Lee, O. Kwon, Y. H. Kim, Protecting entanglement from decoherence using weak measurement and quantum measurement reversal, Nature Physics 8 (2) (2011) 117-120.

[19] G. G. Gillett, R. B. Dalton, B. P. Lanyon, M. P. Almeida, M. Barbieri, G. J. Pryde, J. L. O'Brien, K. J. Resch, S. D. Bartlett, A. G. White, Experimental feedback control of quantum systems using weak measurements, Phys. Rev. Lett. 104 (2010) 080503.

[20] P. B. Dixon, D. J. Starling, A. N. Jordan, J. C. Howell, Ultrasensitive beam deflection measurement via interferometric weak value amplification, Phys. Rev. Lett. 102 (2009) 173601.

[21] J. S. Lundeen, C. Bamber, Procedure for direct measurement of general quantum states using weak measurement, Phys. Rev. Lett. 108 (2012) 070402.

[22] S. Wu, State tomography via weak measurements, Sci. Rep. 3 (1) (2013) 1193.

[23] G. S. Thekkadath, L. Giner, Y. Chalich, M. J. Horton, J. Banker, J. S. Lundeen, Direct measurement of the density matrix of a quantum system, Phys. Rev. Lett. 117 (2016) 120401.

[24] C. C. Rulli, M. S. Sarandy, Global quantum discord in multipartite systems, Phys. Rev. A 84 (2011) 042109.

[25] U. Singh, A. K. Pati, Quantum discord with weak measurements, Ann. Phys. 343 (2014) $141-152$.

[26] V. R. Pande, A. Shaji, Minimum disturbance rewards with maximum possible classical correlations, Phys. Lett. A 381 (25) (2017) $2045-2049$.

[27] Y.-K. Wang, T. Ma, H. Fan, S.-M. Fei, Z.-X. Wang, Superquantum correlation and geometry for Bell-diagonal states with weak measurements, Quan. Info. Proc. 13 (2) (2014) 283-297.

[28] T. Li, T. Ma, Y. Wang, S. Fei, Z. Wang, Super quantum discord for X-type states, Int. J. Theor. Phys. 54 (2) (2015) 680-688.

[29] N. Jing, B. Yu, Super quantum discord for general two qubit X states, Quan. Info. Proc. 16 (4) (2017) 99.

[30] F. Mirmasoudi, S. Ahadpour, Dynamics of super quantum discord and optimal dense coding in quantum channels, J. Phys. A: Math. Theor. 51 (34) (2018) 345302.

[31] M. Xiang, J. Jing, Quantum discord and inferior "geometric discord" based on weak measurement in noninertial frames, J. Quantum Inf. Sci. 4 (2014) 54.

[32] P. R. Dieguez, R. M. Angelo, Weak quantum discord, Quan. Info. Proc. 17 (8) (2018) 194.

[33] J. S. Lee, A. K. Khitrin, Projective measurement in nuclear magnetic resonance, App. Phys. Lett. 89 (7) (2006) 074105.

[34] L. Xiao, J. A. Jones, NMR analogues of the quantum Zeno effect, Phys. Lett. A 359 (5) (2006) $424-427$.

[35] T. Xin, S.-J. Wei, J. S. Pedernales, E. Solano, G.-L. Long, Quantum simulation of quantum channels in nuclear magnetic resonance, Phys. Rev. A 96 (2017) 062303.

[36] J. Von Neumann, Mathematical foundations of quantum mechanics, Princeton University Press, 1955.

[37] Y. Ji, B. Deng, J. Hu, Super-quantum discord with weak measurement in circuit QED, Optik 127 (18) (2016) 7180 - 7187.

[38] M. B. Ruskai, S. Szarek, E. Werner, An analysis of completelypositive trace-preserving maps on M2, Linear Alg. Appl. 347 (1) (2002) $159-187$.

[39] L. Gui-Lu, General quantum interference principle and duality computer, Commun. Theor. Phys. 45 (5) (2006) 825-844.

[40] R. R. Ernst, G. Bodenhausen, A. Wokaun, Principles of NMR in One and Two Dimensions, Clarendon Press, 1990.

[41] D. G. Cory, M. D. Price, T. F. Havel, Nuclear magnetic resonance spectroscopy: An experimentally accessible paradigm for quantum computing, Physica D: Nonlinear Phenomena 120 (1) (1998) $82-101$.

[42] A. Mitra, K. Sivapriya, A. Kumar, Experimental implementation of a three qubit quantum game with corrupt source using nuclear magnetic resonance quantum information processor, J. Magn. Reson. 187 (2) (2007) 306 - 313. 
[43] G. M. Leskowitz, L. J. Mueller, State interrogation in nuclear magnetic resonance quantum-information processing, Phys. Rev. A 69 (2004) 052302.

[44] A. Uhlmann, The "transition probability" in the state space of a *-algebra, Rep. Math. Phys. 9 (2) (1976) 273 - 279.

[45] R. Jozsa, Fidelity for mixed quantum states, J. Mod. Optics 41 (12) (1994) 2315-2323.

[46] B. Dakic, Y. O. Lipp, X. Ma, M. Ringbauer, S. Kropatschek, S. Barz, T. Paterek, V. Vedral, A. Zeilinger, C. Brukner, P. Walther, Quantum discord as resource for remote state preparation, Nature Physics 8 (2012) $666-670$.

[47] D. Cavalcanti, L. Aolita, S. Boixo, K. Modi, M. Piani, A. Winter, Operational interpretations of quantum discord, Phys. Rev. A 83 (2011) 032324. 